\title{
Using Complexity and Spectral Analyses of Noninvasive LDF Signals in Patients with Metabolic Syndrome
}

\author{
Hsin Hsiu ${ }^{D},{ }^{1}$ Hsiao-Feng Hu, ${ }^{2}$ and Yaw-Wen Chang $\mathbb{D}^{3}$ \\ ${ }^{1}$ Graduate Institute of Biomedical Engineering, National Taiwan University of Science and Technology, Taipei, Taiwan \\ ${ }^{2}$ Department of Emergency Medicine, Tri-Service General Hospital, National Defense Medical Center, Taipei, Taiwan \\ ${ }^{3}$ Department of Family \& Community Medicine, Tri-Service General Hospital, National Defense Medical Center, Taipei, Taiwan
}

Correspondence should be addressed to Hsin Hsiu; hhsiu@mail.ntust.edu.tw

Received 13 September 2017; Revised 25 December 2017; Accepted 17 January 2018; Published 25 February 2018

Academic Editor: Arturo Buscarino

Copyright (C) 2018 Hsin Hsiu et al. This is an open access article distributed under the Creative Commons Attribution License, which permits unrestricted use, distribution, and reproduction in any medium, provided the original work is properly cited.

\begin{abstract}
This study performed skin-surface measurements with the aim of verifying if complexity and spectral analyses applied to laserDoppler-flowmetry (LDF) signals can be used to discriminate control subjects and patients with metabolic syndrome (MetS) and patients with premetabolic syndrome (pre-MetS). The study participants $(n=142)$ were assigned to three age-matched groups based on the application of MetS criteria. Beat-to-beat analysis was performed to obtain the mean blood flow (DC), and then approximate entropy (ApEn) values for the 10-minute DC sequences (DC_ApEn) were calculated to evaluate the signal complexity. The wavelet transform was applied and yielded periodic oscillations with five characteristic frequency peaks (defined as FR1-FR5) whose relative energy contributions (RECs) were calculated. DC_ApEn was significantly smaller in MetS and pre-MetS (with one or two Mets factors) patients than in controls, whereas there were no significant differences in DC. The REC of FR1 was significantly smaller while the REC of FR4 was significantly larger in pre-MetS than in MetS. These results indicate the presence of significant differences in ApEn and spectral indexes, which may be partly attributable to changes in microcirculatory regulatory activities accompanying the progression of MetS. The present findings may be pertinent to the early detection of the microcirculatory impairments associated with MetS.
\end{abstract}

\section{Introduction}

Metabolic syndrome (MetS) is a cluster of cardiometabolic abnormalities associated with an increased risk of cardiovascular diseases $[1,2]$. MetS is associated with dysfunction of both the macro- and microcirculation, with MetS patients exhibiting both macro- and microvascular impairments $[1,3]$.

Early detection of vascular complications and preventing their progression are important in the management of cardiovascular diseases. At the macrocirculation level, although the pathways linking components of MetS to increased cardiovascular risk remain unknown, various intermediates such as intima-media thickness and arterial stiffness have been proposed [1]. At the microcirculation level, the cutaneous circulation can be considered representative of the microcirculation in most vascular beds [1], and therefore the skin microcirculation has been proposed as a model of systemic microvascular function that could facilitate investigations of the mechanisms underlying cardiovascular diseases [2].
Laser-Doppler-flowmetry (LDF) utilizes the Doppler shift of the laser light reflected from the red blood cells and has the advantages of noninvasive and real-time measurement for investigations of microcirculatory-blood-flow (MBF) responses. Other than the $\mathrm{MBF}$ supply itself, time-domain indexes of beat-to-beat LDF waveforms are suggested to be able to aid the monitoring of the MBF perfusion condition [4, 5]. Spectral LDF indexes are also used to study the activities of different microcirculatory regulatory mechanisms [6-8]. Nonlinear analysis provides information about the variability structure of skin blood-flow oscillations $[9,10]$. For example, complexity analysis (which could reflect the number of interacting mechanisms [9]) of beat-to-beat LDF signals has been used to study the induced MBF response in subjects with certain diseases or following certain external stimulation $[9,11,12]$.

Among the nonlinear analysis methods, approximate entropy (ApEn) is widely used for evaluating complexity of 
data sequence [13]. In the present study, we applied beat-tobeat, spectral, and ApEn analyses to skin-surface LDF signals with the aim of discriminating the following three agematched groups: MetS patients, premetabolic syndrome (preMetS) patients, and control subjects. The present findings may be pertinent to the early detection of impairment of $\mathrm{MBF}$ perfusion accompanying MetS.

\section{Methods}

2.1. Experimental Procedure. The abbreviations used in this study are summarized in "Abbreviations" section. Details of study methods can be referred to previous studies $[4,6,14]$. Measurement was performed on 142 volunteers; details of subjects are listed in Tables 1-3. The study population was recruited at the Health Management Center of Tri-Service General Hospital. The study was reviewed and approved by the institutional review board of Tri-Service General Hospital (TSGHIRB 2-101-05-045). All subjects gave their informed consent before experiments commenced. According to the criteria listed in Table 1, the subjects were assigned into three age-matched groups: Group A (with no less than three MetS factors, MetS); Group B (with one or two MetS factors, preMetS); Group C (with no MetS factor, control).

2.2. Measurements. ECG signals were measured by surface electrodes (lead II, RA-LL) and acquired by a preamplifier (6600, Gould, USA). MBF flux signal was measured by Moor VMS-LDF (VP1 probe; MBF3, Moor Instruments, UK), with a time constant of $0.001 \mathrm{~s}$, a cut-off frequency of $14.9 \mathrm{kHz}$, and a sampling frequency of $40 \mathrm{~Hz}$ (typical waveforms shown in Figure 1) [14]. The LDF probe was placed at the right side for the subjects. Subjects were asked to sit on a chair and to relax and breathe naturally throughout the measurement period. The signals were sampled at $1024 \mathrm{~Hz}$.

In each recording, 10 min data sequence was acquired. Before the LDF measurement, the heart rate (HR), systolic blood pressure (BP), and diastolic BP were measured by using a sphygmomanometer (MG150f, Rossmax). The environmental temperature was within $23-25^{\circ} \mathrm{C}$ during the entire measuring period. The skin-surface temperature (which was monitored by the same LDF probe) stability was considered acceptable when the variation was less than $1.0^{\circ} \mathrm{C}[4,6]$.

2.3. Data Analysis. Details of study methods can be referred to previous studies [11, 12]. The neighboring minimal points were used to define each pulse. For all the pulses in the 10minute sequence, average of their mean values was calculated to define the microcirculatory blood flow (DC). Values of the coefficient of variance of DC for all the pulses within a 10minute LDF data sequence were then calculated (DC_CV).

ApEn analysis was then performed on each 10-minute sequence of DC (DC_ApEn) of the beat-to-beat LDF data sequence. For a sequence $u(n)$, the ApEn (pattern length $m=$ 2 ; criterion of similarity $r=0.2 \times \mathrm{SD}$ ) can be calculated as follows:

$$
\operatorname{ApEn}=\Phi^{m}(r)-\Phi^{m+1}(r),
$$

where

$$
\begin{aligned}
\Phi^{m}(r) \equiv & (N-m+1) \sum_{i=1}^{-1} \sum_{i}^{N-m+1} \ln C_{i}^{m}(r) \\
C_{i}^{m}(r)= & \text { number of } x(j) \\
& \text { such that } d[x(i), x(j)] \leq r /(N-m+1) \\
x(i)= & {[u(i), \ldots, u(i+m-1)], } \\
x(j)= & {[u(j), \ldots, u(j+m-1)], }
\end{aligned}
$$

where $N$ is number of pulses in the beat-to-beat LDF data sequence and $d$ represents difference between elements $[13,15]$.

In the LDF spectrum of human subjects, periodic oscillations with five characteristic frequency peaks falling within the following frequency bands can be noted: $0.0095-0.02$, $0.02-0.06,0.06-0.15,0.15-0.4$, and $0.4-1.6 \mathrm{~Hz}$ (defined as FR1-FR5, resp.). The relative energy contribution (REC) was defined as the ratio between the total energy density within each band and the entire $0.0095-$ to- $-1.6 \mathrm{~Hz}$ spectrum [6].

2.4. Statistics. All statistical analyses were carried out using SPSS, version 13.0. The differences in the fundamental physiological and LDF parameters were assessed using the Kruskal-Wallis test (nonparametric ANOVA). Post hoc multiple comparisons tests were made by Dunn's test. The level of significance was defined as $p<0.05$; all $p$ values were two-sided hypotheses.

\section{Results}

Details of the included subjects are given in Tables 1 and 2 , and the basic physiological parameters in all groups are listed in Table 3. There were no significant differences in age and HR among the three groups, whereas all of the measured physiological parameters were significantly larger in MetS group than in control group, with the exception of HDL-C (which was smaller).

The comparison of LDF beat-to-beat parameters in Figure 2 reveals that DC_ApEn was significantly smaller in Groups A and B than in control group. There were no significant differences in DC or DC_CV.

The comparison of LDF spectral parameters in Figure 3 reveals that the REC of FR1 was significantly smaller while the RECs of FR4 and FR5 were significantly larger in Group $\mathrm{B}$ than in Group A. There was no significant difference in the REC of any band between Groups A and C.

\section{Discussion}

At the macrovascular level, MetS has been characterized by morphological and functional disorders within large vessels [2]. For example, increases in both the peripheral and central blood pressure, pulse wave velocity, and arterial stiffness have been noted in patients with MetS [1]. At the microvascular level, endothelium-dependent dysfunction of the microcirculation has been reported in patients with MetS without hypertension [2]. MetS patients have also been noted to have 
TABLE 1: Number of subjects fulfilled the criteria of metabolic syndrome.

\begin{tabular}{|c|c|c|c|}
\hline \multirow[b]{2}{*}{ Factor criteria } & \multicolumn{3}{|c|}{ Number of subjects } \\
\hline & $\begin{array}{c}\text { MetS } \\
(n=41)\end{array}$ & $\begin{array}{c}\text { Pre-MetS } \\
(n=67)\end{array}$ & $\begin{array}{l}\text { Control } \\
(n=34)\end{array}$ \\
\hline Waist circumference $\geq 90 \mathrm{~cm}$ (male); $\geq 0.80 \mathrm{~cm}$ (female) & 28 & 32 & 0 \\
\hline Blood pressure $\geq 140 / 90 \mathrm{mmHg}$ & 30 & 22 & 0 \\
\hline High-density lipoprotein cholesterol $\leq 0.9 \mathrm{mmol} / \mathrm{L}$ (male), $\leq 1.0 \mathrm{mmol} / \mathrm{L}$ (female) & 34 & 15 & 0 \\
\hline Fasting plasma glucose $\geq 100 \mathrm{mg} / \mathrm{dl}$ & 20 & 5 & 0 \\
\hline Triglycerides: $\geq 100 \mathrm{mg} / \mathrm{dl}$ & 32 & 7 & 0 \\
\hline
\end{tabular}

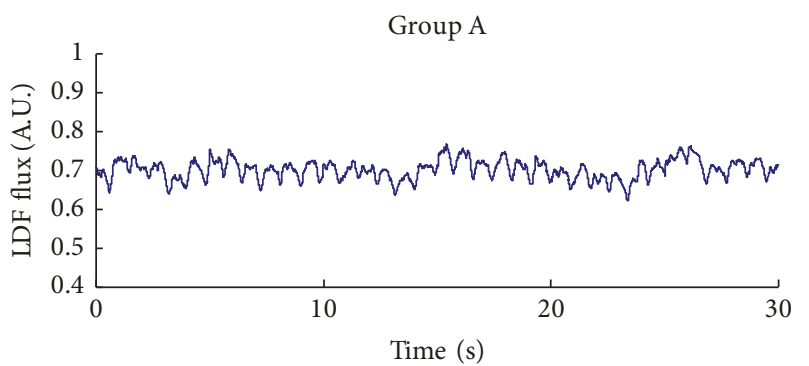

(a)

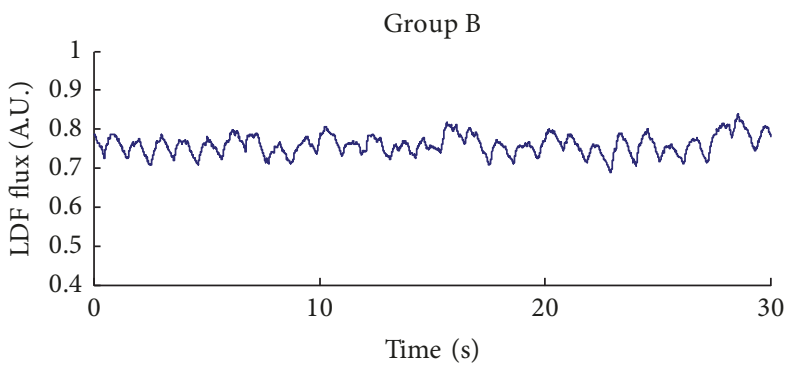

(b)

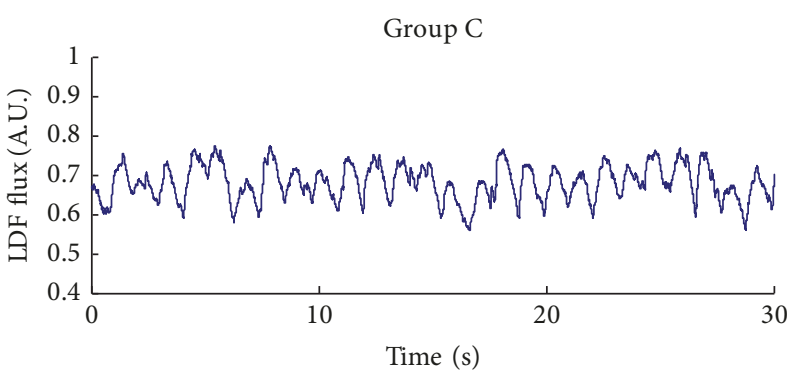

(c)

FIgURE 1: Typical LDF waveforms measured (in arbitrary units) in (a) MetS patients, (b) pre-MetS patients, and (c) control subjects.

TABLE 2: Number of subjects who have chronic conditions.

\begin{tabular}{lccc}
\hline \multirow{2}{*}{ Chronic condition } & \multicolumn{3}{c}{ Number of subjects } \\
& $\begin{array}{c}\text { MetS } \\
(n=41)\end{array}$ & $\begin{array}{c}\text { Pre-MetS } \\
(n=67)\end{array}$ & $\begin{array}{c}\text { Control } \\
(n=34)\end{array}$ \\
\hline Diabetes & 2 & 0 & 0 \\
Cardiovascular disease & 0 & 2 & 1 \\
Hyperlipidemia & 9 & 2 & 2 \\
Hypertension & 4 & 0 & 0 \\
Liver disease & 0 & 13 & 5 \\
Peptic ulcer Disease & 0 & 4 & 0 \\
Renal disease & 0 & 0 & 0 \\
Polycystic ovary syndrome & 0 & 4 & 1 \\
\hline
\end{tabular}

a lower density of functional capillaries [3]. Several possible underlying mechanisms have been proposed; for example, a higher skin microcirculation resistance (possibly induced by lower cutaneous vascular conductance response to ACh and SNP iontophoresis) has implications for the NO pathway, and a decreased production of anti-inflammatory adipokines has been reported in MetS patients [2,3].

4.1. ApEn Index. This study found that DC_ApEn was significantly smaller in Groups A and B than in Group C. A marked reduction in the blood flow associated with both endothelialderived and direct vasodilatory mechanisms has been noted in MetS [3]. In terms of dynamic responses, generalized vasoreactivity impairment has been demonstrated in patients with MetS, even in the absence of type-2 diabetes [2]. Skin microvascular abnormalities in response to ischemia have been observed in MetS patients [16]. It is possible that such a defect in the peripheral blood flow supply reduces the microcirculatory regulatory activity. The number of participating regulatory mechanisms may be decreased or the degrees of freedom of the regulatory activities may be reduced due to an impaired MBF supply. These factors may decrease the complexity in the skin-surface LDF signals and thus decrease the ApEn value. Furthermore, there was a progressive increase in the ApEn values in Groups A-C. This illustrates that the presence of decreased complexity in the skin-surface LDF beat-to-beat index may accompany the progression of MetS. 
TABLE 3: Fundamental physiological parameters (quantity or mean \pm standard deviation) of subjects.

\begin{tabular}{|c|c|c|c|c|}
\hline Parameters & $\begin{array}{c}\text { MetS } \\
(n=41)\end{array}$ & $\begin{array}{c}\text { Pre-MetS } \\
(n=67)\end{array}$ & $\begin{array}{l}\text { Control } \\
(n=34)\end{array}$ & Statistical significance ${ }^{\dagger}$ \\
\hline Gender (male/female) & $27 / 14$ & $22 / 45$ & $15 / 19$ & \\
\hline Age (years) & $44.5 \pm 10.6$ & $48.4 \pm 12.9$ & $42.7 \pm 10.5$ & \\
\hline Heart rate (beats/minute) & $73.3 \pm 9.2$ & $73.3 \pm 9.5$ & $71.1 \pm 8.2$ & \\
\hline $\operatorname{BMI}\left(\mathrm{kg} / \mathrm{m}^{2}\right)$ & $26.8 \pm 3.5$ & $24.4 \pm 3.8$ & $22.3 \pm 3.0$ & $\mathrm{AC}$ \\
\hline Waist circumference $(\mathrm{cm})$ & $89.3 \pm 8.2$ & $82.9 \pm 8.9$ & $79.6 \pm 9.1$ & $\mathrm{AC}$ \\
\hline Systolic blood pressure (mm-Hg) & $126.2 \pm 12.5$ & $119.8 \pm 14.5$ & $106.1 \pm 11.3$ & $\mathrm{AC}, \mathrm{BC}$ \\
\hline Diastolic blood pressure $(\mathrm{mm}-\mathrm{Hg})$ & $85.7 \pm 7.5$ & $79.5 \pm 10.4$ & $70.4 \pm 6.9$ & $\mathrm{AC}, \mathrm{BC}$ \\
\hline $\mathrm{HDL}-\mathrm{C}(\mathrm{mg} / \mathrm{dl})$ & $39.8 \pm 10.0$ & $57.1 \pm 9.5$ & $60.3 \pm 13.7$ & $\mathrm{AB}, \mathrm{AC}$ \\
\hline Fasting glucose (mg/dl) & $98.4 \pm 8.8$ & $90.3 \pm 6.5$ & $85.1 \pm 5.1$ & $\mathrm{AB}, \mathrm{AC}$ \\
\hline Triglyceride (mg/dl) & $237.6 \pm 168.1$ & $101.0 \pm 42.2$ & $93.2 \pm 32.4$ & $\mathrm{AB}, \mathrm{AC}$ \\
\hline
\end{tabular}

${ }^{\dagger}$ Significant difference between groups $(p<0.05)$. A: MetS, B: pre-MetS, and C: control.

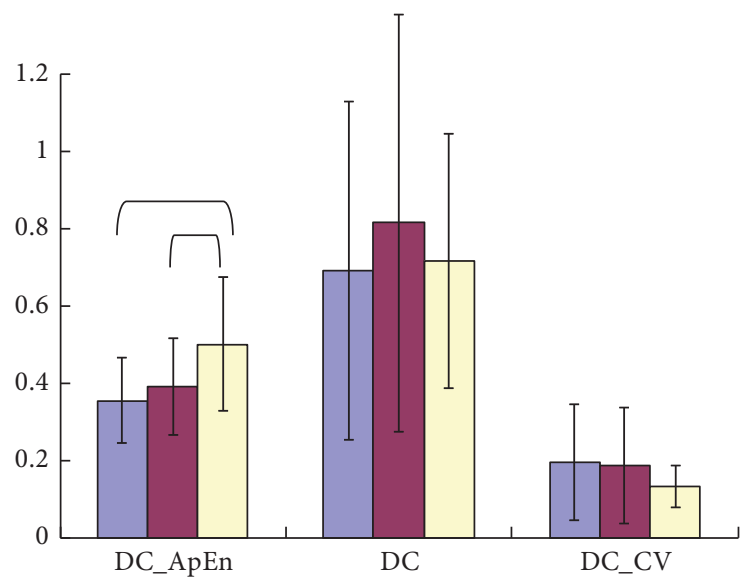

$\square$ A
$\square$ B
$\square$ C

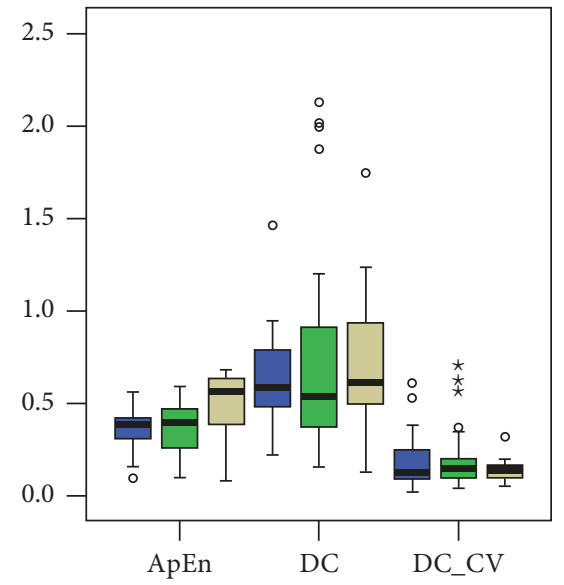

$\square$ Group A

Group B

Group C

Figure 2: Comparison of LDF beat-to-beat parameters (mean \pm SD). “ $\neg$ ” indicates $p<0.05$. DC_ApEn was significantly smaller in Groups A and B than in control group. There were no significant differences in DC or DC_CV. The circles indicate far values and “*” indicates far out values.
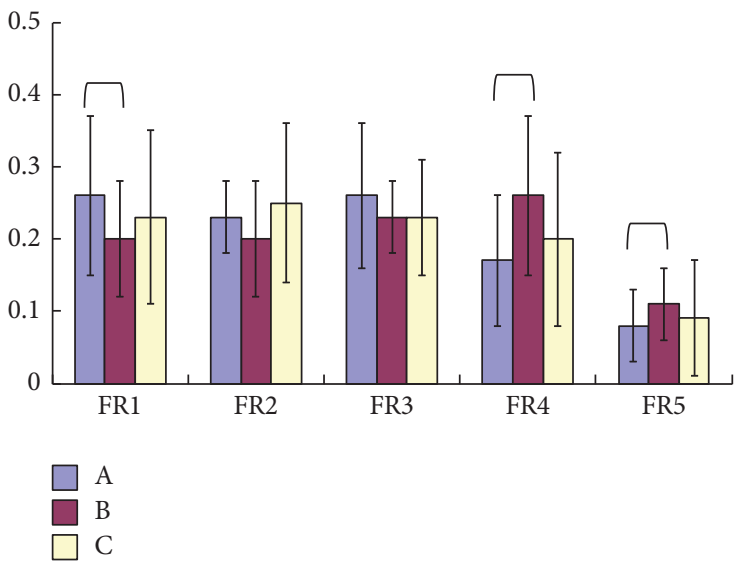

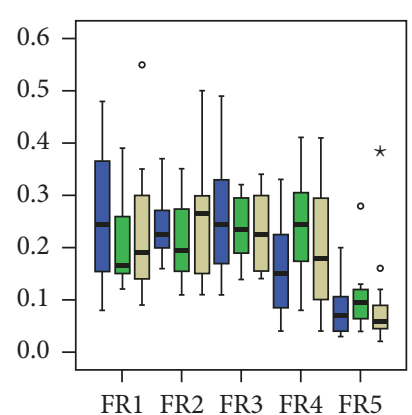

Group A

Group B

Group C

FIGURE 3: Comparison of LDF spectral parameters (mean \pm SD). “ $\neg$ ” indicates $p<0.05$. The REC of FR1 was significant smaller while the RECs of FR4 and FR5 were significantly larger in Group B than in Group A. There were no significant differences between Groups A and C. The circles indicate far values and “*” indicates far out values. 
4.2. DCCV. Beat-to-beat cardiovascular variability parameters such as $\mathrm{HR}$ variability and BP variability are widely used to monitor cardiovascular regulatory activity. Similarly, variability indexes for the MBF supply might also be suitable for monitoring the microcirculatory regulatory activities in local vascular beds. Changes in MBF variability parameters have been suggested to be related to the adjustment effort of the cardiovascular system when it is stimulated in different ways $[17,18]$. Significant differences in MBF variability parameters have been found between normal control subjects and patients with various types of diseases, including stroke and diabetes $[4,5]$. However, the present study found no significant intergroup differences in DCCV, which illustrates that the ApEn index is better than the DCCV index for distinguishing between the present MetS patients and control subjects. A possible explanation for this difference is DCCV being related to the extent that $\mathrm{MBF}$ perfusion varies in the presence of microcirculatory regulation, whereas DC_ApEn could reflect the complexity in the MBF fluctuation patterns of MetS patients under changes in certain specific types of microcirculatory regulatory activity; for example, it is possible that DC_ApEn is affected more by the local microcirculatory regulatory activities that change the elastic or morphological properties of the local vascular beds.

4.3. Spectral Indices. LDF spectral analysis has been widely used in studies of the microcirculatory regulatory activities in local vascular beds $[6,7]$. Calculating LDF spectral indexes may help to further identify the possible changes in the microcirculatory regulatory activities in MetS patients. The present study found significant differences in the RECs of several frequency bands of the LDF signals; for example, the REC of FR1 was significantly smaller in Group pre-MetS than in Group MetS. The vascular endothelium plays a key role in the pathogenesis of microangiopathy and neuropathy by producing compounds such as nitric oxide, endothelin, and prostacyclin [3]. Endothelium-dependent dysfunction of the microcirculation has been reported in patients with MetS without hypertension [2]. It has been suggested that changes in the REC of FR1 are associated with endothelial regulatory functions in the microcirculation [7]. The differences in REC of FR1 noted between Groups MetS and pre-MetS could be at least partly attributable to endothelial function being more severely impaired in the former group. An impaired endothelial function may decrease the amount and types of vasoactive agents secreted, reduce the degrees of freedom for local microcirculatory endothelial regulation, and decrease the complexity in MBF fluctuations, thereby leading to decreases in the ApEn value in Group MetS.

The REC of FR4 was significantly smaller in Group MetS than in Group pre-MetS. It has been suggested that changes in the REC of FR4 are associated with baroreflex regulatory functions in the microcirculation [7]. Both SBP and DBP were significantly smaller in control group than in the other groups. It is possible that, during the early stage of MetS progression, the baroreflex regulatory functions can be activated to compensate the effects of an increased BP. This could have resulted in the REC of FR4 being slightly larger (although nonsignificantly) in Group pre-MetS than in Group control.
The induced effect of the increase in BP could have lasted longer and been larger in Group MetS than in Group preMetS. There could be a gradual reduction in the ability of the baroreflex to adjust the BP value, hence resulting in the REC of FR4 being significantly smaller in Group MetS than in Group pre-MetS. This could partly account for the difference in the changes in the REC of FR4 between Groups MetS and pre-MetS.

4.4. Discussion in the Present Indices. The present results for ApEn values may help to differentiate control subjects from patients with MetS and pre-MetS, while those for spectral indexes may help to differentiate between MetS and preMetS patients. Changes in the ApEn values precede those in the beat-to-beat LDF indexes themselves, and so the present findings may be pertinent to the early detection of the impairment of MBF perfusion associated with MetS.

Few previous studies have focused on the effects on the complexity in MBF perfusion accompanying MetS. However, the effect of aging has been widely discussed. For example, it has been reported that the complexity in MBF is significantly lower among those aged 75-85 years than those aged 65-75 years [10], which can be partly attributed to vascular impairment associated with aging. This means that the mechanism underlying the decreased ApEn value in the present Group MetS (e.g., vascular impairment) may be similar to vascular aging effects. Another study found that increased complexity in the skin-surface LDF beat-to-beat index paralleled the disease progression of diabetes. Impairment of the MBF perfusion condition in diabetic patients (perhaps due to impaired endothelial function caused by hyperglycemia, oxidative stress, and dyslipidemia) may result in further impairment of the ability to maintain homeostasis of the MBF supply, which may in turn have led to the increases in complexity and ApEn values measured in the present diabetic patients [19]. It is possible that, compared with diabetic patients, such impairment in the MBF perfusion condition had not yet occurred in the MetS patients, which is also supported by the absence of significant differences in DC values between the present groups. The change in the ability to maintain homeostasis of the MBF supply was less severe in MetS patients, and hence the change in the complexity in the MBF signals differed between MetS and diabetic patients.

\section{Conclusion}

The following conclusions can be drawn from the results obtained in this study.

(i) The presence of decreased complexity in the beatto-beat LDF index sequence may be at least partly attributable to changes in the microcirculatory regulatory activities or impairment of the homeostasis mechanism of MBF perfusion.

(ii) The spectral analysis of the LDF signals revealed that the differences in the ApEn values could be related to the changes in the endothelial function and baroreflex activity accompanying the progression of MetS. 


\section{Abbreviations}

MBF: Microcirculatory blood flow

Mets: $\quad$ Metabolic syndrome

Pre-Mets: Premetabolic syndrome

LDF: $\quad$ Laser Doppler flowmetry

AO: $\quad$ Arteriolar openings

ApEn: Approximate entropy

BP: Blood pressure

HR: Heart rate

DC: $\quad$ Mean microcirculatory blood flow

DCCV: Coefficient of variance of DC sequence

DC_ApEn: Approximate entropy of DC sequence

FR1-FR5: Five frequency bands in LDF spectrum

REC: $\quad$ Relative energy contribution.

\section{Conflicts of Interest}

The authors declare that they have no conflicts of interest.

\section{Acknowledgments}

The authors would like to thank the Ministry of Science and Technology (MOST 104-2221-E-011-170-MY3) and National Defense Medical Center (TSGH-C102-014) for partial support of this work.

\section{References}

[1] S. Czernichow, J. R. Greenfield, P. Galan et al., "Macrovascular and microvascular dysfunction in the metabolic syndrome," Hypertension Research, vol. 33, no. 4, pp. 293-297, 2010.

[2] G. Walther, P. Obert, F. Dutheil et al., "Metabolic syndrome individuals with and without type 2 diabetes mellitus present generalized vascular dysfunction: Cross-sectional study," Arteriosclerosis, Thrombosis, and Vascular Biology, vol. 35, no. 4, pp. 1022-1029, 2015.

[3] A. I. Vinik, M. Nevoret, C. Casellini, and H. Parson, "Neurovascular function and sudorimetry in health and disease," Current Diabetes Reports, vol. 13, no. 4, pp. 517-532, 2013.

[4] H. Hsiu, H. F. Hu, G. S. Wu, and F. C. Hsiao, "Characteristics in the beat-to-beat laser-Doppler waveform indices in subjects with diabetes," Clinical Hemorheology and Microcirculation, vol. 57, no. 4, pp. 375-384, 2014.

[5] C.-T. Chen, H. Hsiu, J.-S. Fan, F.-C. Lin, and Y.-T. Liu, "Complexity analysis of beat-to-beat skin-surface laser-doppler flowmetry signals in stroke patients," Microcirculation, vol. 22, no. 5, pp. 370-377, 2015.

[6] H.-F. Hu, H. Hsiu, C.-J. Sung, and C.-H. Lee, "Combining laser-Doppler flowmetry measurements with spectral analysis to study different microcirculatory effects in human prediabetic and diabetic subjects," Lasers in Medical Science, vol. 32, no. 2, pp. 327-334, 2017.

[7] A. Stefanovska, M. Bracic, and H. D. Kvernmo, "Wavelet analysis of oscillations in the peripheral blood circulation measured by laser Doppler technique," IEEE Transactions on Biomedical Engineering, vol. 46, no. 10, pp. 1230-1239, 1999.

[8] P.-C. Sun, C.-S. Chen, C.-D. Kuo et al., "Impaired microvascular flow motion in subclinical diabetic feet with sudomotor dysfunction," Microvascular Research, vol. 83, no. 2, pp. 243-248, 2012.
[9] A. P. Blaber, R. L. Bondar, F. Stein et al., "Complexity of middle cerebral artery blood flow velocity: Effects of tilt and autonomic failure," American Journal of Physiology-Heart and Circulatory Physiology, vol. 273, no. 5, pp. H2209-H2216, 1997.

[10] F. Liao, D. W. Garrison, and Y.-K. Jan, "Relationship between nonlinear properties of sacral skin blood flow oscillations and vasodilatory function in people at risk for pressure ulcers," Microvascular Research, vol. 80, no. 1, pp. 44-53, 2010.

[11] H. Hsiu, W.-C. Hsu, C.-L. Hsu, J.-G. Bau, C.-T. Chen, and Y.S. Liu, "Complexity analysis of the microcirculatory-blood-flow response following acupuncture stimulation," Microvascular Research, vol. 89, pp. 34-39, 2013.

[12] H. Hsiu, W.-C. Hsu, C.-L. Hsu, and J.-G. Bau, "Microcirculatory complexity responses to the application of skin-surfacecontacting pressure stimulation around normal blood pressure," Journal of Medical and Biological Engineering, vol. 34, no. 2, pp. 164-171, 2014.

[13] S. M. Pincus, "Approximate entropy as a measure of system complexity," Proceedings of the National Acadamy of Sciences of the United States of America, vol. 88, no. 6, pp. 2297-2301, 1991.

[14] H. Hsiu, W.-C. Hsu, Y.-F. Wu, C.-L. Hsu, and C.-Y. Chen, "Differences in the skin-surface laser doppler signals between polycystic ovary syndrome and normal subjects," Microcirculation, vol. 21, no. 2, pp. 124-130, 2014.

[15] R. B. Panerai, "Complexity of the human cerebral circulation," Philosophical Transactions of the Royal Society A: Mathematical, Physical \& Engineering Sciences, vol. 367, no. 1892, pp. 1319-1336, 2009.

[16] L. G. Kraemer-Aguiar, C. M. Laflor, and E. Bouskela, "Skin microcirculatory dysfunction is already present in normoglycemic subjects with metabolic syndrome," Metabolism, vol. 57, no. 12, pp. 1740-1746, 2008.

[17] H. Hsiu, C.-L. Hsu, and M.-Y. Jan, "Relations between beat-tobeat microcirculatory blood flow and variations therein," Photomedicine and Laser Surgery, vol. 28, no. 6, pp. 785-792, 2010.

[18] H. Hsiu, S.-M. Huang, and T.-L. Hsu, "Evaluation of the function of arteriolar opening by variability in microcirculatory blood flow following angiotensin II administration in rats," Biorheology, vol. 47, no. 3-4, pp. 239-253, 2010.

[19] H. Hsiu, C. L. Hsu, H.-F. Hu, F.-C. Hsiao, and S.-H. Yang, "Complexity analysis of beat-to-beat skin-surface laser-Doppler signals in diabetic subjects," Microvascular Research, vol. 93, pp. 9-13, 2014. 


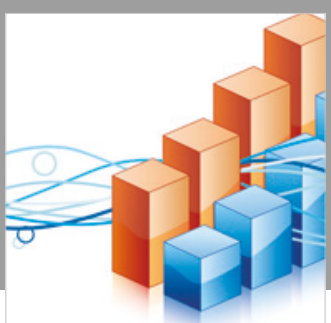

Advances in

Operations Research

\section{-n-m}
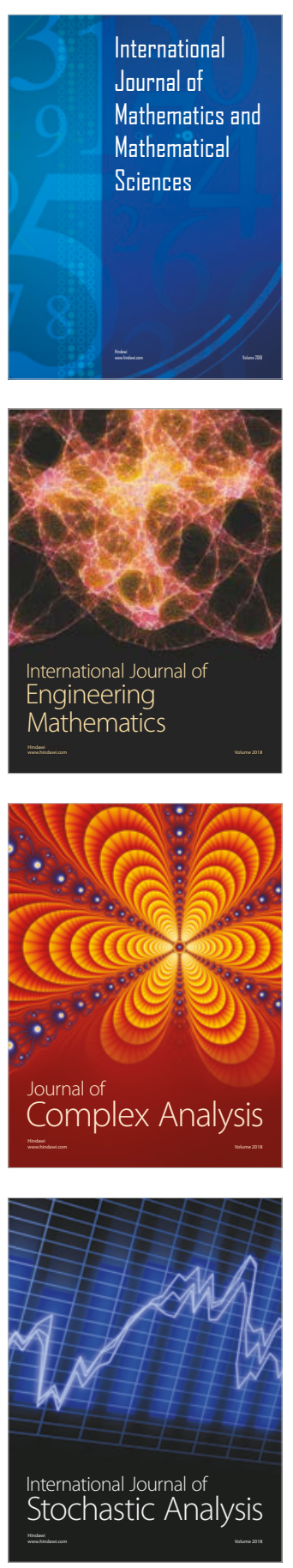
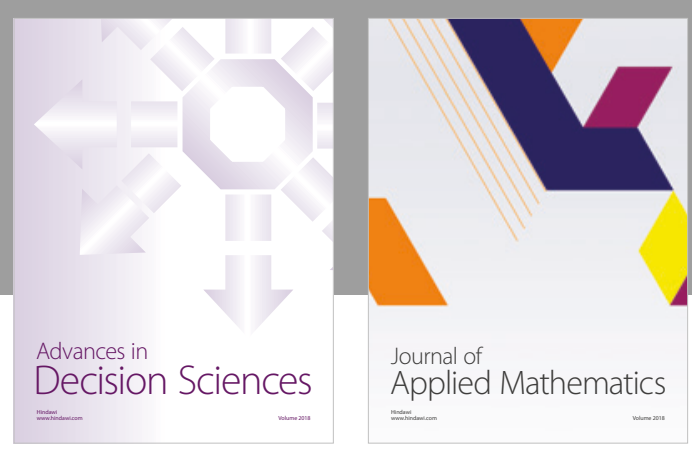

Journal of

Applied Mathematics
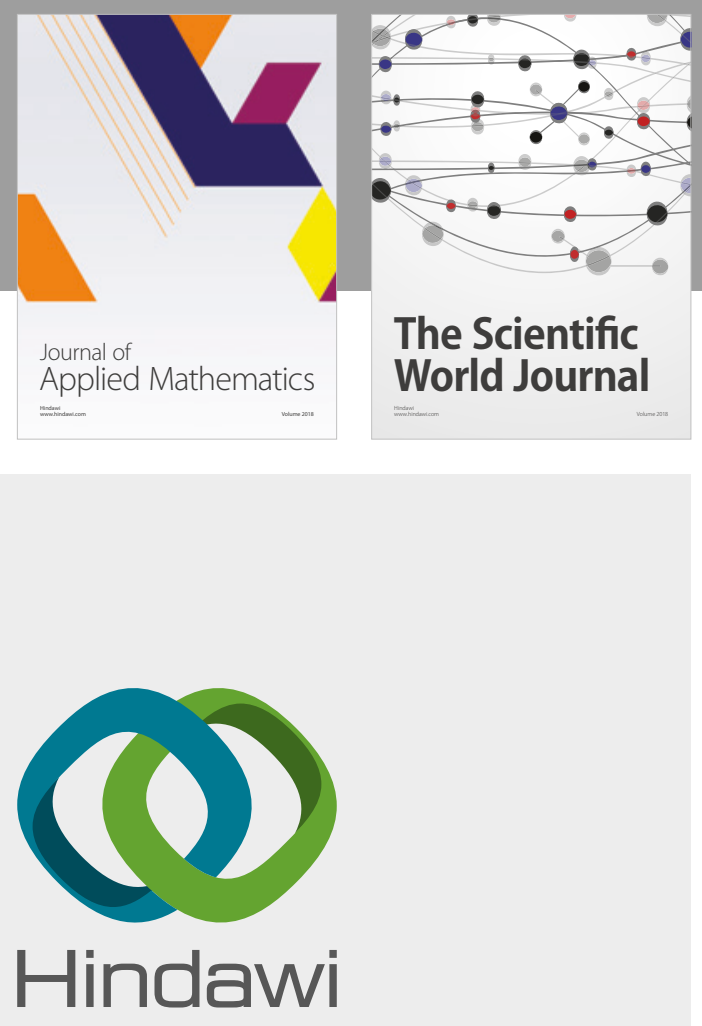

Submit your manuscripts at

www.hindawi.com

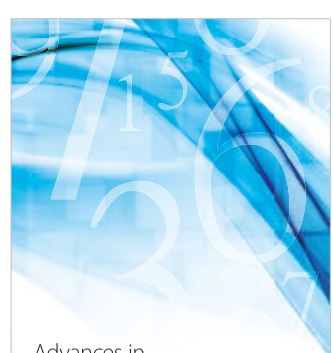

Advances in
Numerical Analysis
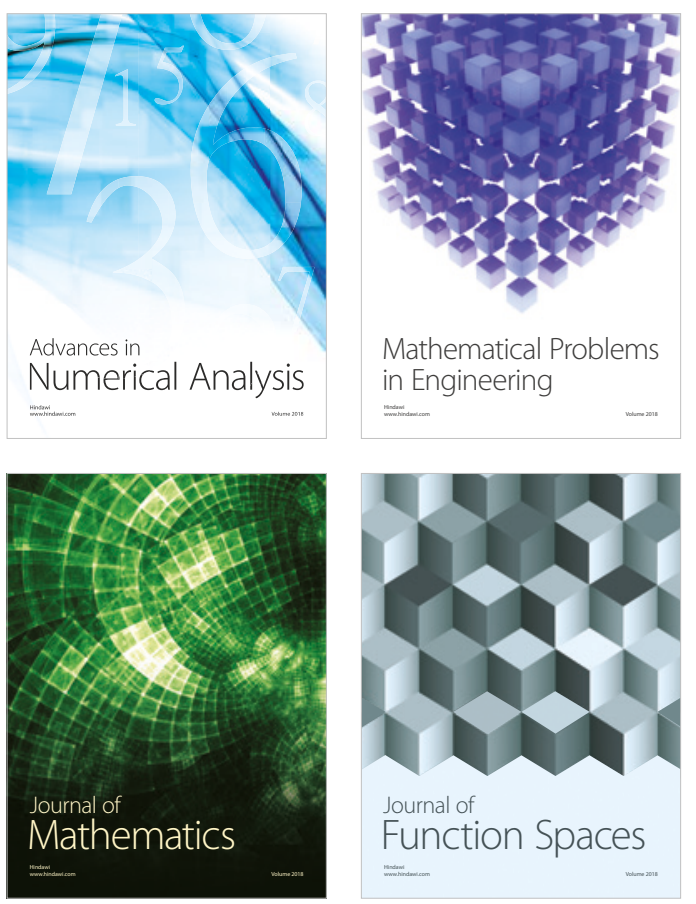

Mathematical Problems in Engineering

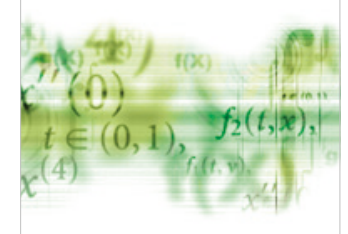

International Journal of

Differential Equations

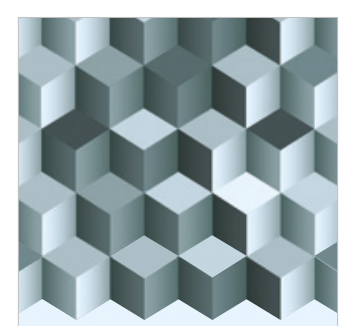

Journal of

Function Spaces
The Scientific

World Journal

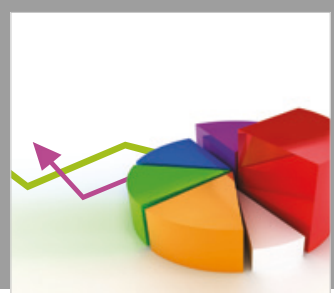

Journal of

Probability and Statistics
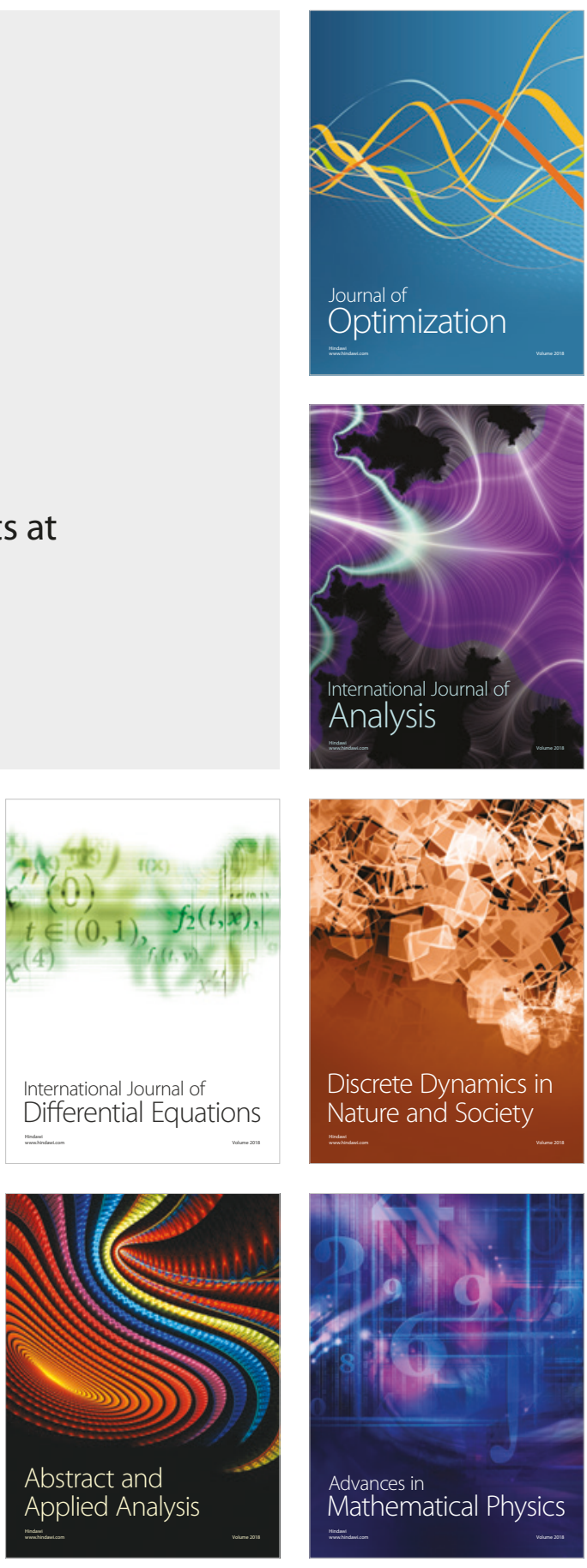